\author{
W. Nemer, K. Rebbas \& F. Krouchi
}

\title{
Découverte de Cypripedium calceolus (Orchidaceae) au Djurdjura (Algérie), nouvelle pour l'Afrique du Nord
}

\begin{abstract}
Nemer, W., Rebbas, K. \& Krouchi, F.: Découverte de Cypripedium calceolus (Orchidaceae) au Djurdjura (Algérie), nouvelle pour l'Afrique du Nord. — Fl. Medit. 29: 207-214. 2019. — ISSN: 1120-4052 printed, 2240-4538 online.

Discovery of Cypripedium calceolus (Orchidaceae) in Djurdjura (Algeria), news for North Africa. - Cypripedium calceolus L. (Orchidaceae) native to Eurasia is considered one of the flagship plant species of nature conservation. Because of its wide range, this species could be considered a taxon of minor concern, near threatened, vulnerable, endangered or critically endangered.

This orchid was discovered in the Djurdjura, in north-central Algeria. Ecological notes are also provided. This is the first report of this species in North Africa. This discovery will enrich the Algerian orchid in general and that of Kabylia in particular.

Key words: flora, floristics, Orchid, North Africa.
\end{abstract}

\section{Introduction}

La famille des orchidées est l'une des familles de plantes à graines les plus riches en espèces. Il comprend environ 880 genres comprenant plus de 25000 espèces dans le monde (Cribb \& al. 2003).

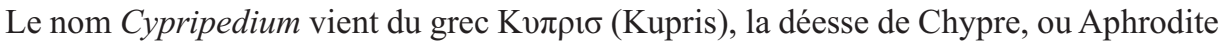
(Vénus), la déesse de l'amour des Romains et des Grecs, et du grec $\pi \varepsilon \delta$ เov (pedion), plante du pied. Un des noms français du Cypripedium est Sabot de Vénus, d'où le nom du genre. L'espèce "calceolus" signifie en latin "petite chaussure".

Bien que mondialement reconnue comme un des principaux point-chauds de biodiversité végétale (Médail \& Quezel 1997 ; Médail \& Myers 2004), la région méditerranéenne demeure méconnue, en particulier sur ses rives sud et est. L'ensemble de montagnes du littoral algéro-tunisien dénommé «Kabylies-Numidie-Kroumirie» ne fait pas exception avec une forte diversité végétale et un fort taux d'endémisme (Véla \& Benhouhou 2007).

Malgré l'engouement énorme qu'ont suscité les orchidées européennes (cf. Delforge 2016), l'orchidoflore des rives sud de la Méditerranée demeure méconnue. 
En Afrique du Nord, Cypripedium calceolus n'est pas citée dans l'index de Dobignard \& Chatelain (2010-2013). De même aucune flore ou catalogue d'Algérie (Battandier 18881890; Battandier \& Trabut 1905; Maire 1952-1987; Quézel \& Santa 1962-1963) ni même de Tunisie (Bonnet \& Barrate 1896; Pottier-Alapetite 1979-1981; Le Floc'h \& al. 2010; Guittonneau 2011), ni aussi au Maroc (Fennane \& Ibn Tattou 1998, 2005; Fennane \& al. 2007) ne signale ce taxon. Cette orchidée n'est pas signalée dans l'Euro+Med PlantBase (Euro+Med 2006).

Le présent travail vient faire le point sur la découverte de Cypripedium calceolus dans le Djurdjura et son écologie.

\section{Contexte de la découverte}

\section{Cypripedium calceolus L.}

Nomencl. ref.: Sp. P1.: 951. 1753 (WCSP 2019)

Avec une quarantaine d'individus groupés en cinq touffes, occupant une aire inférieur à $25 \mathrm{~m}^{2}$, le Sabot de Vénus a été découvert en plein floraison le 7 juin 2019 par l'un de nous (W.N.) dans une station située au sud-ouest du col de Tirourda sur une pente orientée ouest à une altitude de $1485 \mathrm{~m}\left(36.4516^{\circ} \mathrm{N}\right.$ et $\left.04.3294^{\circ} \mathrm{E}\right)$ à l'occasion d'un inventaire floristique des rochers et des falaises du Djurdjura (Kabylie, Algérie). Le 29 juin, une visite des deux auteurs (W.N. \& K.R.) a permis d'observer des individus de cette orchidée en extrême fin de floraison, complètement fanées (Fig. 1 et 2). Un spécimen d'herbier collecté a été déposé dans l'herbier officiel de l'école nationale supérieure agronomique (ENSA) d'Alger le 18.9.2019. Le nouveau site algérien est représenté par une zone semi-ombragée située entre des ilots de reboisement de Cedrus atlantica. Le tableau 1 présente une liste de la flore inventoriée dans la station de C. calceolus.

Dans d'autres pays, cette orchidée occupe des habitats forestiers en Eurasie qui ont un couvert forestier semi-ouvert permettant à un peu de lumière de l'atteindre. Sa présence en Mordovie (Russie centrale) est confinée dans divers types d'habitats : forêts de feuillus, forêts de conifères et forêts mixtes. Plus un habitat de prairies pures (Baumann \& Künkele 1982 ; Bournérias \& Prat 2005 ; Antonelli \& al. 2009 ; García \& al. 2010 ; Khapugin \& al. 2017).

En Europe, C. calceolus est une calcicole bien connue qui pousse dans des sols alcalins ou rarement neutres. En Europe centrale, il pousse dans les forêts de conifères et en bordure de forêts mixtes de feuillus, souvent dans des sites plats ou en pente, jusqu'à une altitude de $2000 \mathrm{~m}$ ou plus. À certains endroits, il forme de grandes colonies avec des plantes individuelles portant de nombreuses pousses (KS - RBG 2019).

\section{Répartition géographique}

Le nouveau site de Cypripedium calceolus en Algérie est situé à plus de $500 \mathrm{~km}$ au sud des sites pyrénéens.

En Europe, on trouve cette orchidée depuis le niveau de la mer au nord jusqu'à plus de 2000 m d'altitude dans les Alpes. En France, elle est toujours rare, de la Lorraine et de la Haute-Marne aux Alpes, très rare dans les Pyrénées et les grands Causses ; disparu en 


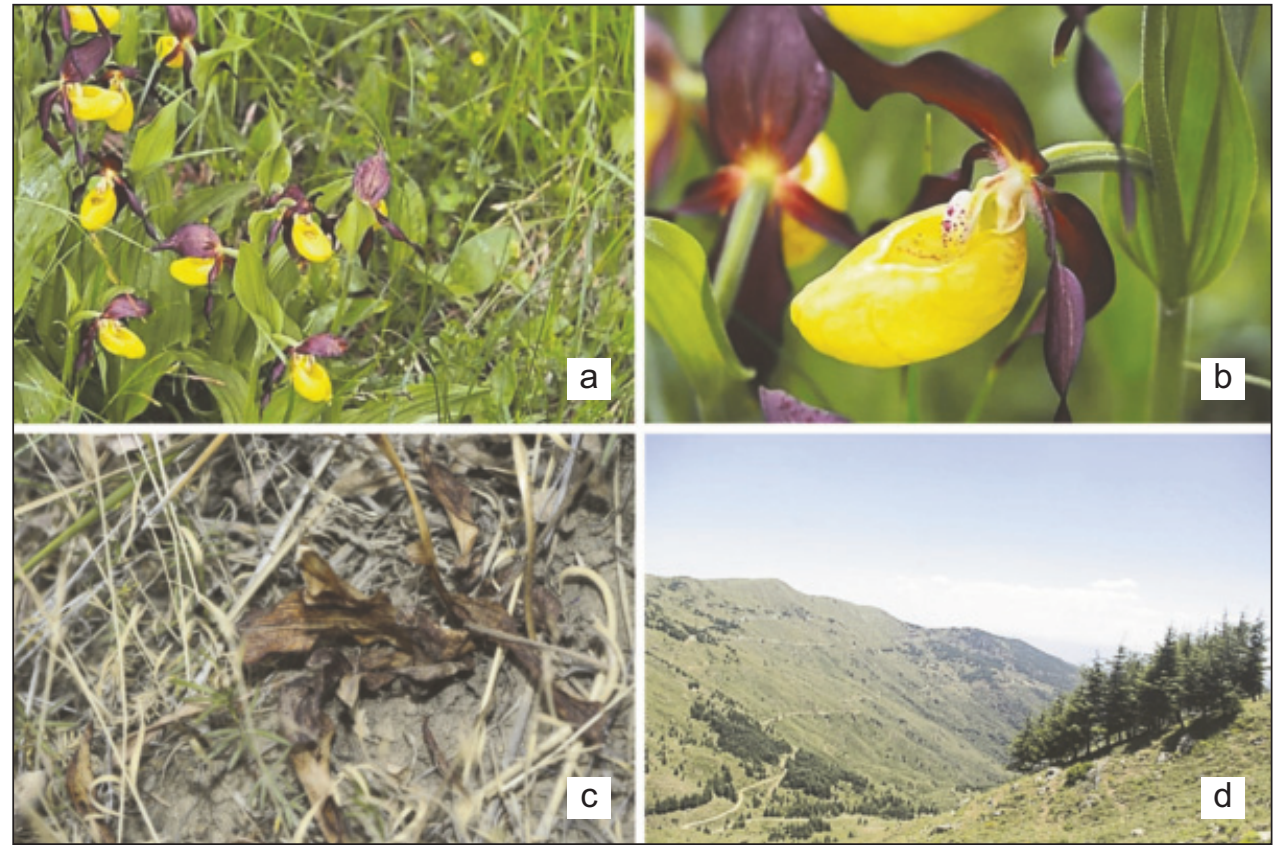

Fig. 1. Illustration de Cypripedium calceolus dans le Djurdjura: a \& b) individus en fleurs, 7.6.2019 (photos W. Nemer); c) individu fané; d) site de la découverte, 29.6.2019 (photo K. Rebbas).

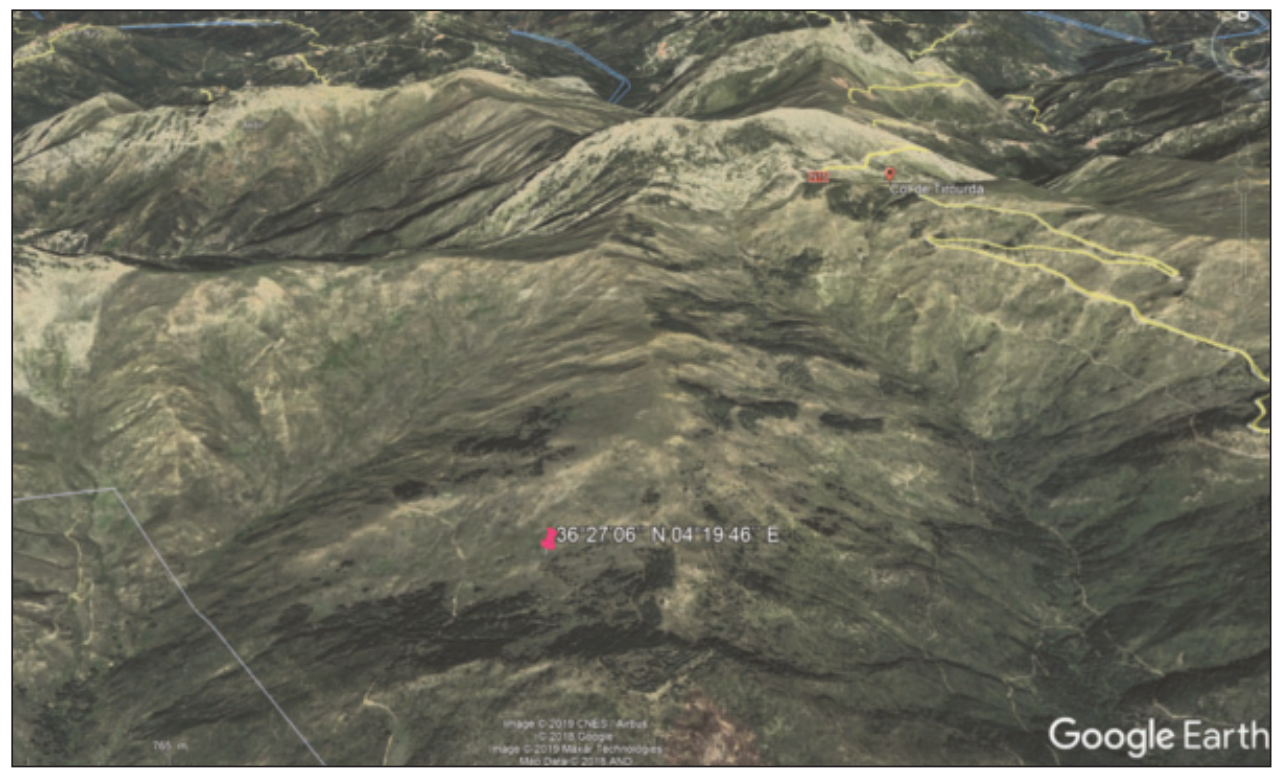

Fig. 2. Localisation géographique de la station nord-africaine de Cypripedium calceolus dans le Djurdjura, au sud-ouest du col de Tirourda. 
Tableau 1. Liste de la flore inventoriée dans la station de C. calceolus.

\begin{tabular}{|c|c|}
\hline \multicolumn{2}{|c|}{ Nom scientifique } \\
\hline Allium tenuiflorum Ten. & Dianthus vulturius Guss. \& Ten \\
\hline $\begin{array}{l}\text { Acinos alpinus subsp. meridionalis (Nyman) P.W. } \\
\text { Ball }\end{array}$ & Epipactis tremolsii $\mathrm{Pau}$ \\
\hline Aegilops triuncialis L. & Genista tricuspidata Desf. \\
\hline Allium fontanesii J. Gay & Himantoglossum hircinum (L.) Spreng. \\
\hline $\begin{array}{l}\text { Ampelodesmos mauritanicus (Poir.) T. Durand \& } \\
\text { Schinz }\end{array}$ & Inula montana L. \\
\hline Anacamptis pyramidalis (L.) Rich. & $\begin{array}{l}\text { Juniperus communis subsp. hemisphaerica (C. Presl) } \\
\text { Nyman }\end{array}$ \\
\hline Anisantha rubens (L.) Nevski & Jurinea humilis (Desf.) DC. \\
\hline Anthyllis montana $\mathrm{L}$. & Lomelosia crenata (Cirillo) Greuter \& Burdet \\
\hline Anthyllis vulneraria L. & Mantisalca salmantica (L.) Briq. \& Cavill. \\
\hline Aphanes floribunda (Murb.) Rothm. & Orchis anthropophora (L.) All. \\
\hline Armeria plantaginea All. & Orchis olbiensis Gren. \\
\hline Asperula hirsuta Desf. & Origanum vulgare subsp. glandulosum (Desf.) Ietsw. \\
\hline Astragalus armatus subsp. numidicus (Murb.) Tietz & Plantago mauritanica Boiss. \& Reut. \\
\hline Bartsia trixago L. & Poa bulbosa L. \\
\hline Briza maxima $\mathrm{L}$. & Quercus ilex subsp. ballota (Desf.) Samp. \\
\hline Bupleurum spinosum Gouan & Rosa canina $\mathrm{L}$. \\
\hline Carduus macrocephalus Desf. & Sedum acre $\mathrm{L}$. \\
\hline Carthamus atractyloides (Pomel) Greuter & Sedum album $\mathrm{L}$. \\
\hline Catananche caerulea $\mathrm{L}$. & Sedum caeruleum $\mathrm{L}$. \\
\hline Cedrus atlantica (Endl.) Carrière & Sedum sediforme (Jacq.) Pau \\
\hline Centaurea pubescens Willd. & Silene coelirosa (L.) Godr. \\
\hline Crataegus laciniata Ucria & $\begin{array}{l}\text { Thymus munbyanus subsp. ciliatus (Desf.) Greuter \& } \\
\text { Burdet }\end{array}$ \\
\hline Crucianella angustifolia $\mathrm{L}$. & Thymus willdenowii Boiss. \\
\hline Dactylis glomerata $\mathrm{L}$. & Trifolium arvense $\mathrm{L}$. \\
\hline Dactylorchis battandieri Raynaud & Trifolium stellatum $\mathrm{L}$. \\
\hline Dianthus serralatus subsp. eu-serrulatus Maire & Trisetum flavescens (L.) P. Beauv. \\
\hline
\end{tabular}

Belgique et au Luxembourg (Bournérias \& Prat 2005). Cypripedium calceolus est répandu dans les Pays-Bas, Amérique du Nord, Eurasie et Japon. En Europe, il vient du sud-ouest jusqu'aux Pyrénées. Sur la péninsule, il ne pousse qu'à Huesca et à Barcelone (Bañares \& al. 2004). La Suède continentale est une région qui compte les plus grandes populations européennes (Antonelli \& al. 2009). En Angleterre, Cypripedium calceolus n'a jamais été aussi répandu et a été surexploité au point d'être presque éteint vers la première moitié du XXe siècle. Il s'est accroché sur un site et a depuis été propagé et réintroduit avec succès. Ailleurs, il est extrêmement rare (par exemple au Danemark) ou éteint (par exemple en Grèce), et atteint des populations relativement importantes (par exemple en Pologne et en Autriche).

En raison de sa large aire de répartition, cette espèce d'Eurasie pourrait être considérée comme un taxon faisant l'objet d'une préoccupation mineure, quasi menacée, vulnérable, en voie de disparition ou gravement en danger (Khapugin \& al. 2017). Bien que C. calceolus soit inclus dans la liste rouge mondiale de l'UICN avec la caté- 
gorie Préoccupation mineure (LC), il s'agit d'une des espèces d'orchidées les plus menacées d'Europe (Kull 1999). Cette orchidée est inscrite sur plusieurs listes rouges nationales comme menacée : - Régionalement éteinte au Luxembourg. - En danger critique d'extinction en Bulgarie, en Serbie et au Royaume-Uni. - En danger en Croatie, en République tchèque, en Hongrie, en Russie et en Espagne. - Vulnérable en Autriche, Biélorussie, Danemark, France, Allemagne, Lituanie, Slovaquie et Suisse. - Presque menacé en Finlande et en Norvège. - Préoccupation mineure en Suède (Rankou \& Bilz 2014). - En danger critique d'extinction en Mordovia (Khapugin \& al. 2017).

\section{Conservation de Cypripedium calceolus}

Les orchidées pantoufles sont communes dans les Amériques, ainsi qu'en Europe et dans les régions tempérées et asiatiques, au sud de l'Himalaya. Cependant, au début des années 1980, C. calceolus, pantoufle de la dame britannique, était réduit à un individu à floraison unique dans la nature. La collecte excessive par les amateurs d'orchidées et la disparition d'habitats convenables ont vu leur nombre diminuer jusqu'à l'extinction. En 1983, Sir Robert et Lady Sainsbury ont donc financé un projet dans l'unité de micropropagation de Kew pour tenter d'améliorer les techniques de culture d'orchidées sauvages dans des conditions artificielles (KS - RBG 2019).

L'expérience de culture en plein air de plants de Cypripedium calceolus L. ex vitro en République Tchèque a donné les premiers résultats positifs (Obdržálek 2009).

Des analyses d'extinction ont montré que pour une population d'une vingtaine d'individus (caractéristiques de nombreuses localités européennes), il n'y a aucune chance de survie si deux plantes seulement sont enlevées à environ cinq ans d'intervalle, un niveau d'agression qui n'est probablement pas atypique (Terschuren 1999). Cependant, pour aggraver encore la situation en matière de conservation, la pollinisation limite également le succès de reproduction des orchidées (Antonelli \& al. 2009).

Il est protégé au niveau national dans la plupart des pays (France, Hongrie, par exemple) et la collecte de l'espèce est interdite (par exemple, en Lituanie). De nombreuses populations sont incluses dans des sites Natura 2000 et d'autres formes d'aires protégées. La protection des sites et une gestion appropriée sont essentielles (Rankou \& Bilz 2014).

\section{Conclusion}

La poursuite de ce travail visera l'exploration botanique d'autres stations de la région de la Kabylie.

Pour la conservation durable du site jusqu'alors connu de Cypripedium calceolus au Djurdjura, de sévères mesures de protection semblent indispensables. Il convient d'analyser l'utilisation actuelle de la zone ainsi que les menaces éventuelles (surpâturage, incendies, réchauffement climatique) de la petite population, d'envisager la gestion future et les mesures de conservation par culture semblent être intéressantes à un stade ultérieur. 
Il est important de classer cette orchidée dans la liste de plantes non cultivées et protégées figurant dans le décret exécutif algérien (J.O.R.A. 2012) et que nos prochaines explorations botaniques dans le parc national de Djurdjura apportent davantage d'informations concernant la phytosociologie et la dynamique de la population de C. calceolus pour son évaluation dans la liste rouge de l'UICN.

\section{Remerciements}

L'exploration à l'origine de cette découverte n'aurait pas été possible sans la complicité et l'efficacité de nos compagnons de terrain Mustapha Lateb, Massi Madouche et Youcef Rebbas. Les auteurs tiennent à remercier les relectures pour leurs remarques et leurs commentaires afin d'améliorer cet article.

\section{Références}

Antonelli, A., Dahlberg, C. J., Carlgren, K. H. I. \& Appelqvist, T. 2009: Pollination of the Lady's slipper orchid (Cypripedium calceolus) in Scandinavia - taxonomic and conservational aspects. - Nordic J. Bot. 27: 266-273. http://dx.doi.org/10.1111/j.1756-1051.2009.00263.x

Averyanov, L.V. 2000: The genus Cypripedium (Orchidaceae) in Russia. - Lindleyana 15(4): 197-221.

Bañares, Á., Blanca, G., Güemes, J., Moreno, J. C. \& Ortiz, S. (eds) 2004: Atlas y Libro Rojo de la Flora Vascular Amenazada de España. - Madrid.

Battandier, J. A. 1888-1890: Flore d'Algérie: Ancienne flore d'Alger transformée (Dicotylédones). - Alger.

— \& Trabut, L. C. 1905: Flore analytique et synoptique de l'Algérie et de la Tunisie. - Alger. https://doi.org/10.5962/bhl.title.10806

Baumann, H. \& Künkele, S. 1982: Die wildwachsenden Orchideen Europas. - Stuttgart.

Bonnet, E. \& Barratte, G. 1896: Catalogue raisonné des plantes de la Tunisie. - Paris. https://doi.org/10.5962/bhl.title.11048

Bournérias, M. \& Prat, D. (eds) 2005: Les Orchidées de France; Belgique et Luxembourg, ed. 2. - Mèze.

Delforge, P. 2016 : Orchidées d'Europe, d'Afrique du Nord et du Proche Orient. 4e édition revue et augmentée. - Neuchâtel.

Dobignard, A. \& Chatelain, C. 2010-2013: Index synonymique de la flore d'Afrique du Nord. Genève.

Conti, F., Manzi, A. \& Pedrotti F. 1997: Liste Rosse Reginali delle Piante d'Italia. - Camerino.

FFH-Directive 2019: Council Directive 92/43/EEC of 21 May 1992 on the conservation of natural habitats and of wild fauna and flora. https://eurlex.europa.eu/LexUriServ/LexUriServ.do?uri=CONSLEG:1992L0043:20070101:EN:PDF [dernier accès 12/07/2019]. https://doi.org/10.1017/cbo9780511610851.039

Cribb, P. J., Kell, S. P., Dixon, K. W. \& Barrett, R. L. 2003: Orchid conservation: a global perspective. - Pp. 1-24 in: Dixon, K. W., Kell, S. P., Barrett, R. L. \& Cribb, P. J. (eds), Orchid conservation. - Kota Kinabalu.

Euro+Med 2006: Euro+Med PlantBase - the information resource for Euro-Mediterranean plant diversity. Published on the Internet http://ww2.bgbm.org/EuroPlusMed/ [dernier accès 12/07/2019].

Fennane, M. \& Ibn Tattou, M. 1998: Catalogue des plantes vasculaires rares, menacées ou endémiques du Maroc. - Bocconea 8: 5-243. 
— \& - 2005: Flore vasculaire du Maroc, inventaire et chorologie. - Trav. Inst. Sci. Univ. Mohammed V, Sér. Bot. 37: 1-202.

—, - Ouyahya, A. \& El Oualidi, J. 2007: Flore pratique du Maroc. - Trav. Inst. Sci. Univ. Mohammed V, Sér. Bot. 38:1-636.

García, M. B., Goñi, D. \& Guzmán, D. 2010: Living at the Edge: Local versus Positional Factors in the Long-Term Population Dynamics of an Endangered Orchid. - Conserv. Biol. 24(5): 12191229. http://dx.doi.org/10.1111/j.1523-1739.2010.01466.x

Guittonneau, G. G. 2011: La flore et la végétation de la Tunisie méridionale. - Voy. Etud. Soc. Bot. France (27 mars -3 avril 2008): 283-358.

J.O.R.A. 2012: Décret exécutif du 18 janvier 2012, complétant la liste des espèces végétales non cultivées et protégées. - J. Off. Rép. Algérienne, 3-12.

Hultén, E. \& Fries M. 1986: Atlas of North European vascular plants north of the tropic of cancer, 1. - Königstein

KS - RBG (Kew Science - Royal Botanic Gardens). 2019: Cypripedium calceolus L. Plants of the World online. http://www.plantsoftheworldonline.org/taxon/urn:lsid:ipni.org:names:320700-2 [dernier accès 01/10/2019]

Khapugin, A. A., Chugunov, G. G. \& Vargot, E. A. 2017: Cypripedium calceolus (Orchidaceae) in Central Russia: a case study for its populations in two protected areas in the Republic of $\begin{array}{lllll}\text { Mordovia } & \text { (Russia). } & - & \text { Lankesteriana } & \text { 17(3): }\end{array}$ http://dx.doi.org/10.15517/lank.v17i3.31577

Kull, T. 1999: Cypripedium calceolus L. -J. Ecol. 87: 913-924. http://dx.doi.org/10.1046/j.13652745.1999.00407.x

Le Floc'h, E., Boulos, L. \& Véla, E. 2010: Catalogue synonymique commenté de la flore de Tunisie. - Tunis.

Maire, R. 1952-1987: Flore de l'Afrique du Nord (Maroc, Algérie, Tunisie, Tripolitaine, Cyrénaïque et Sahara), 1-16. - Paris.

Medail, F. \& Myers, N. 2004: Mediterranean Basin. - Pp. 144-147 in: Mittermeier, R. A., Robles Gil, P., Hoffmann, M., Pilgrim, J, Brooks, T., Goettsch Mittermeier, C., Lamoreux, J. \& da Fonseca

G. A. B. (eds) Hotspots revisited: Earth's Biologically Richest and Most Endangered Terrestrial Ecoregions. - Monterrey, Washington.

— \& Quezel, P. 1997: Hot-spot analysis for conservation of plants biodiversity in the Mediterranean Basin. - Ann. Missouri Bot. Gard. 84: 112-127. https://doi.org/10.2307/2399957

Munby, G. 1847: Flore de l'Algérie ou catalogue des plantes indigènes. - Paris.

Obdržálek, J. 2009: Cultivation of Cypripedium calceolus L. ex vitro seedlings in outdoor conditions. - Hort. Sci. (Prague) 36(4): 162-170. https://doi.org/10.17221/4/2009-hortsci

Pottier-Alapetite, M. 1979-1981: Flore de la Tunisie, Angiospermes-Dicotylédones, 1-2. -Tunis.

Terschuren, J. 1999: Action plan for Cypripedium calceolus in Europe. - Nature Environ. 100. - Strasbourg

Quézel, P. \& Santa, S. 1962-1963: Nouvelle flore de l'Algérie et des régions désertiques méridionales, 1-2. - Paris.

Rankou, H. \& Bilz, M. 2014: Cypripedium calceolus, Lady's Slipper Orchid. - IUCN Red List of Threatened $\quad$ Species. http://dx.doi.org/10.2305/IUCN.UK.2014-1.RLTS. T162021A43316125.en_[dernier accès 01/10/2019]

Vela, E. \& Benhouhou, S. 2007: Évaluation d'un nouveau point chaud de biodiversité végétale dans le Bassin méditerranéen (Afrique du Nord). - Comptes-Rendus Biol. 330: 589-605. http://dx.doi.org/10.1016/j.crvi.2007.04.006 
WCSP, 2019: Cypripedium calceolus L., Sp. Pl.: 951 (1753). http://wcsp.science.kew.org/namedetail.do?name_id=53948 [dernier accès 01/10/2019]

Adresses des auteurs :

Walid Nemer ${ }^{1}$, Khellaf Rebbas ${ }^{2}$ \& Fazia Krouchi ${ }^{1}$,

${ }^{1}$ Laboratoire de Production, Amélioration et Protection des Végétaux et des Denrées alimentaires, Faculté des Sciences Biologiques et des Sciences Agronomiques, Université Mouloud Mammeri, Tizi-Ouzou, Algérie. Courriel: walidnemer51@gmail.com; krfaz@yahoo.com

${ }^{2}$ Département des Sciences de la Nature et de la Vie, Faculté des Sciences, Université Mohamed Boudiaf, M'Sila, Algérie. Laboratoire d'Agro-Biotechnologie et de Nutrition en Zones arides et semi arides / Equipe de Gestion des Ressources naturelles et environnement, Université Ibn Khaldoun, Tiaret, Algérie. Courriel: rebbas.khellaf@gmail.com; khellaf.rebbas@univ-msila.dz 